Supporting information for

\title{
Immobilized Titanium (IV) ion affinity chromatography contributes to efficient proteomics analysis of cellular nucleic acid binding proteins
}

Huiyu Wang ${ }^{a, \dagger}{ }^{\dagger}$ Liqiang Qian ${ }^{b, \dagger}$, Zhi Shang ${ }^{c,}{ }^{\dagger}$, Zeyuan Wang a, Yan Zhang ${ }^{d}$, Chengxi

$$
\mathrm{Cao}^{\mathrm{e}} \text {, Hua Xiao }{ }^{\mathrm{a}, *}
$$

a State Key Laboratory of Microbial Metabolism, Joint International Research Laboratory of Metabolic \& Developmental Sciences, School of Life Sciences and Biotechnology, Shanghai Jiao Tong University, Shanghai, 200240, China.

${ }^{b}$ Department of Shanghai Lung Cancer Center, Shanghai Chest Hospital, Shanghai Jiao Tong University, Shanghai, 200030, China.

c Institute of Clinical Immunology, Department of Liver Diseases, Central Laboratory, ShuGuang Hospital Affiliated to Shanghai University of Chinese Traditional Medicine, Shanghai, 200021, China.

dSchool of Pharmacy, Shanghai Jiao Tong University, Shanghai, 200240, China

e Department of Instrument Science and Engineering, School of Electronic Information and Electrical Engineering, Shanghai Jiao Tong University, Shanghai 200240, China

${ }^{*}$ Corresponding Author:

Prof. Dr. Hua Xiao, Email address: huaxiao@sjtu.edu.cn

tThese authors contributed equally as first authors to this work. 


\section{This supporting information contains:}

Supporting Experimental Methods

Figure S1. DNA and RNA adsorption and elution efficiency using $\mathrm{Ti}^{4+}$-IMAC in vitro

Figure S2. Agarose gel electrophoresis results of NAs-protein complex before and after $\mathrm{Ti}^{4+}-\mathrm{IMAC}$ capture using $(\mathrm{A})$ chemical reagent and $(\mathrm{B})$ organic solvent as cell lysis buffer

Figure S3. Agarose gel electrophoresis of NAs-protein adducts before and after decrosslinking.

Figure S4. Volcano plots of GO annotated NABPs with (A) $0.1 \%$ FA (B) $0.2 \%$ FA (C) $0.5 \%$ FA (D) $1 \%$ FA cross-linking compared with total cell lysate

Figure S5. The number and proportion of GO annotated RBPs, DBPs and Non NABPs obtained with different organic solvents and dosage

Figure S6. Principal component analysis of the quantification data of proteins in different groups

Figure S7. Pearson coefficients of the quantitative MS results

Figure S8. Cell components analysis of protein extracted by (A) organic solvent extraction and $(\mathrm{B}) \mathrm{Ti}^{4+}-\mathrm{IMAC}$ capture method

Figure S9. Ten of the most significant over-represented (A) molecular function and (B) biology process obtained from 1255 non-NABP proteins in organic solvent extraction and 767 non-NABP proteins in $\mathrm{Ti}^{4+}-$ IMAC method

Figure S10. Distribution of GO annotated DBPs, RBPs and NABPs and number of GO annotated NABPs with different foldchange $\left(\mathrm{Ti}^{4+}-\mathrm{IMAC}\right.$ capture method /total 
cell lysate).

\section{Supplemental Tables}

Supplementary Table S1. Proteins obtained by using different concentration of formaldehyde. (XLSX)

Supplementary Table S2. Proteins obtained by different precipitation methods. (XLSX)

Supplementary Table S3. Comparison of different extraction strategies for NAs-protein complexes. (XLSX)

Supplementary Table S4. Lung cancer NABPome analysis. (XLSX)

\section{Supporting Information}

\section{Experimental methods}

\section{MS sample preparation.}

For MS analysis, NABPs were first de-cross-linked from NAs-protein complexes by heat at $65^{\circ} \mathrm{C}$ for $6 \mathrm{~h}$. Then digested by using Filter-aided sample preparation (FASP) approach. In brief, $50 \mu \mathrm{g}$ proteins were reduced with DTT $(20 \mathrm{mM})$ at $60{ }^{\circ} \mathrm{C}$ for $60 \mathrm{~min}$ and alkylated with $(50 \mathrm{mM})$ iodoacetamide at room temperature in the dark for $40 \mathrm{~min}$. The sample solution was transferred to $10 \mathrm{kDa}$ ultrafiltration tube (Vivacon, Berlin, Germany) at $4{ }^{\circ} \mathrm{C}$ and centrifuged $12000 \mathrm{~g}$ for $20 \mathrm{~min}$. Then the sample was washed with $50 \mathrm{mM} \mathrm{NH} \mathrm{NHCO}_{3}$ for three times and further digested with trypsin at $37{ }^{\circ} \mathrm{C}$ for $16 \mathrm{~h}$. Digested peptides were collected by centrifugation at $12000 \mathrm{~g}$ at $4{ }^{\circ} \mathrm{C}$ for $20 \mathrm{~min}$, and then washed two times with 50 $\mathrm{mM} \mathrm{NH}{ }_{4} \mathrm{HCO}_{3}$. Obtained peptides were dried and stored at $-80^{\circ} \mathrm{C}$ for subsequent experiments. 


\section{Protein identification and label-free quantification}

LTQ Orbitrap XL mass spectrometer coupled with Easy-nLC 1000

The peptides were first loaded onto a $2 \mathrm{~cm} \times 75 \mu \mathrm{m}$ fused silica capillary column packed in-house with Reprosil-Pur 200 C18-AQ (3 $\mu \mathrm{m}, 200 \AA$, Dr. Maisch), then trapped peptides were eluted to a $22 \mathrm{~cm} \times 75 \mu \mathrm{m}$ fused silica capillary column packed in-house with Reprosil-Pur 120 C18-AQ (3 $\mu \mathrm{m}, 120 \AA$, Dr. Maisch). Peptides were eluted from columns by Mobile phase A and B. Mobile phase $A$ was $0.1 \%$ formic acid in water, and mobile phase $B$ was $0.1 \%$ formic acid in acetonitrile. The total flow rate was $600 \mathrm{~nL} / \mathrm{min}$, and the gradient was performed as follows: $5 \% \mathrm{~B}$ (0-5 min), 5-22\% B (6-95 min), 22-32\% B (96- $110 \mathrm{~min}), 32-90 \%$ B (111-113 min), 90\% B (114-120 min). Detailed instrument parameters are shown in Table S1.

Orbitrap Q-Exactive Plus mass spectrometer coupled with Easy-nLC 1000

Peptides digests were separated by a C18 analytical column $(50 \mu \mathrm{m} \times 25 \mathrm{~cm}, 2$ $\mu \mathrm{m}$ C18, $300 \AA$, Phenomenex) then eluted by mobile phase. The mobile phase consisted of buffer $A(0.1 \%$ formic acid in water) and buffer $B(0.1 \%$ formic acid in $95 \%$ acetonitrile). A flow rate of $300 \mathrm{~nL} / \mathrm{min}$ and $120 \mathrm{~min}$ gradient was applied as follows: 5\% B (0-5 min), 5-22\% B (6-95 min), 22-32\% B (96-110 min), 32-90\% B (111-113 min), 90\% B (114-120 min). Detailed instrument parameters are shown in the Table S1.

Quantitative data analysis was performed by MaxQuant (v1.6.1.2) using raw data files of MS/MS spectra searched against the UniProtKB human database (release 2017_11_28, 20244 entries), an initial search was set at a precursor mass window of $6 \mathrm{ppm}$. The search followed an enzymatic cleavage rule of 
Trypsin/P and allowed maximal two missed cleavage sites and a mass tolerance of $20 \mathrm{ppm}$ for fragment ions. Carbamidomethylation of cysteines was defined as fixed modification, while protein $\mathrm{N}$-terminal acetylation and methionine oxidation were defined as variable modifications for database searching. Maximum FDR, calculated by employing a reverse database strategy, was set at $1 \%$ for peptides and proteins. Label-free quantification was performed by Intensity-based absolute quantification (iBAQ). Protein expression from each type of cell lines was compared using normalized iBAQ value, based on at least two unique peptides, and data processing was aligned. The 2 folds cut-off was set up for the screening of differentially expressed proteins.

Table S1. MS instrument parameters

\begin{tabular}{|c|c|c|c|c|}
\hline \multirow{4}{*}{} & \multicolumn{2}{|c|}{ QE Plus } & \multicolumn{2}{c|}{ LTQ Orbitrap XL } \\
\cline { 2 - 5 } & source type & NSI & source type & NSI \\
\cline { 2 - 5 } & capillary temp (C) & 275 & capillary temp (C) & 275 \\
\cline { 2 - 5 } MS1 & spray voltage (KV) & 2 & source voltage (KV) & 2.4 \\
\cline { 2 - 5 } & Polarity & positive & Polarity & positive \\
\cline { 2 - 5 } & Scan range (m/z) & 350 to 1500 & Scan range (m/z) & 350 to 2000 \\
\cline { 2 - 5 } & Resolution & 70,000 & Resolution & 30,000 \\
\cline { 2 - 5 } & AGC target & $3 \times 10^{6}$ & FTMS full AGC Target & $5 \times 10^{5}$ \\
\hline \multirow{4}{*}{ MS2 } & Resolution & 17500 & Resolution & $/$ \\
\cline { 2 - 5 } & AGC target & $1 \times 10^{5}$ & ion trap MSn AGC Target & $1 \times 10^{4}$ \\
\cline { 2 - 5 } & maximum IT & $45 \mathrm{~ms}$ & ion trap MSn max ion time & $100 \mathrm{~ms}$ \\
\cline { 2 - 5 } & cisolation window & $1.6 \mathrm{~m} / \mathrm{z}$ & isolation window & $2 \mathrm{~m} / \mathrm{z}$ \\
\cline { 2 - 5 } & Scan range & 200 to 2000 & Scan range & 150 to 2000 \\
\cline { 2 - 5 } & activation type & HCD & activation type & CID \\
\cline { 2 - 5 } & NCE & 28 & coll. Energy & 35 \\
\cline { 2 - 5 } & intensity threshold & $2.2 \times 10^{5}$ & & \\
\hline
\end{tabular}




\begin{tabular}{|l|l|l|l|l|}
\hline & dynamic exclusion & $30 \mathrm{~s}$ & exclusion duration & $60 \mathrm{~s}$ \\
\hline
\end{tabular}




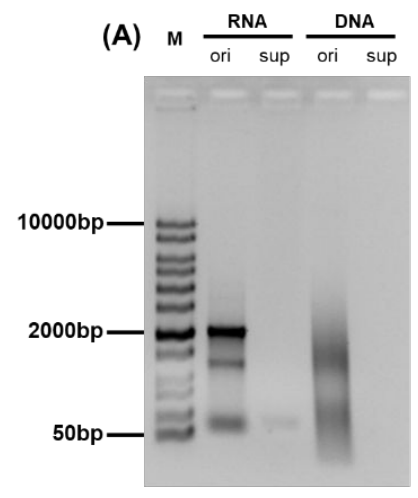

(B)

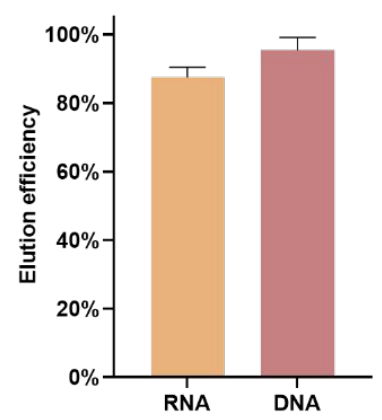

Figure S1. (A) Agarose gel electrophoresis of DNA and RNA before and after $\mathrm{Ti}^{4+}-$ IMAC capture. (B) DNA and RNA elute efficiencies from $\mathrm{Ti}^{4+}-\mathrm{IMAC}$. Ori: before $\mathrm{Ti}^{4+}-$ IMAC capture, sup: after $\mathrm{Ti}^{4+}-$ IMAC capture 

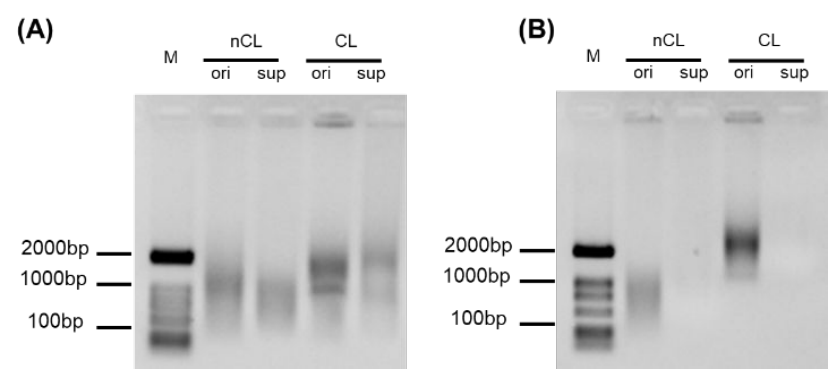

Figure S2. (A) Agarose gel electrophoresis of NAs-protein complex extraction before and after $\mathrm{Ti}^{4+}-\mathrm{IMAC}$ capture using classical lysate. $\mathrm{M}$ is the DNA marker. (B) Agarose gel electrophoresis of NAs-protein complexes extraction before and after $\mathrm{Ti}^{4+}-$ IMAC capture using organic solvent pretreatment. $\mathrm{M}$ is the DNA marker

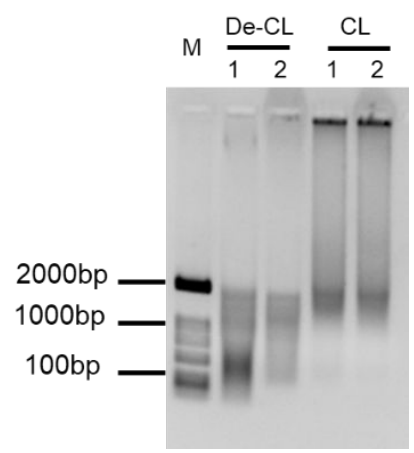

Figure S3. Agarose gel electrophoresis of NAs-protein adducts before and after decrosslinking 
(A) Formaldehyde_0.1\%

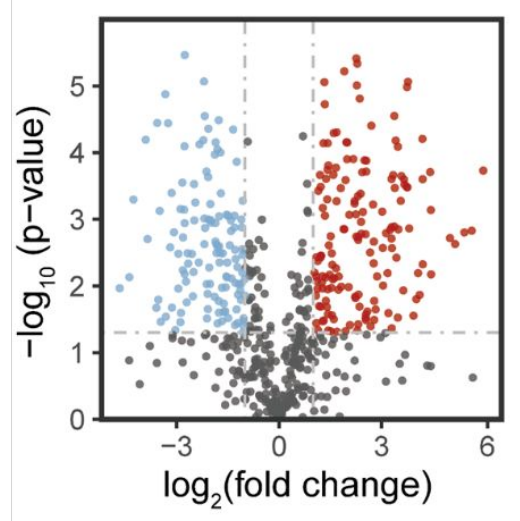

(C) Formaldehyde_0.5\%

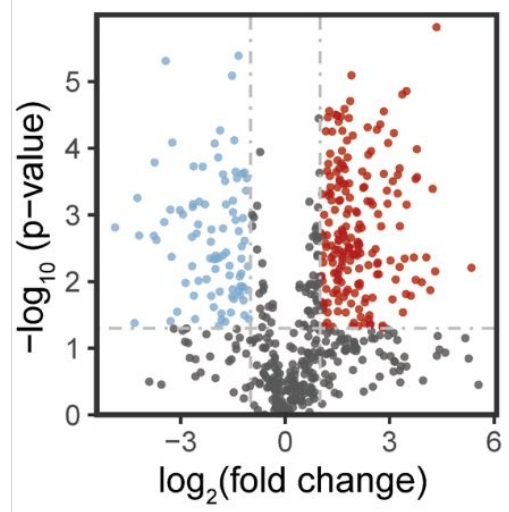

(B) Formaldehyde_0.2\%

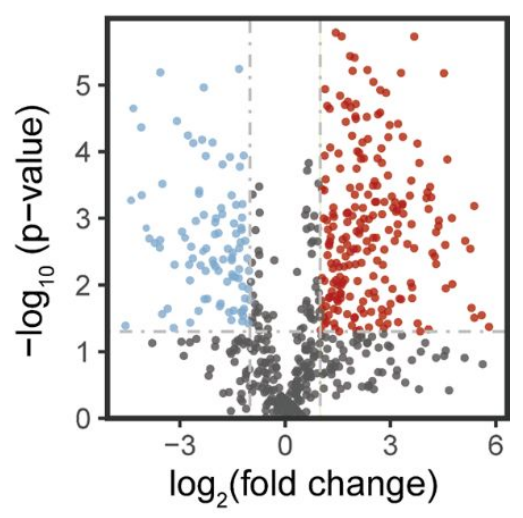

(D) Formaldehyde_1\%

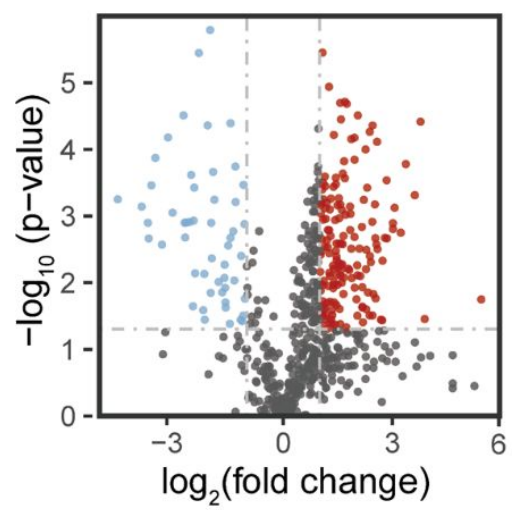

Figure S4. Volcano plots of GO annotated NABPs with (A) $0.1 \%$ FA (B) $0.2 \%$ FA (C) $0.5 \% \mathrm{FA}$ (D) $1 \% \mathrm{FA}$ cross-linking compared with total cell lysate 


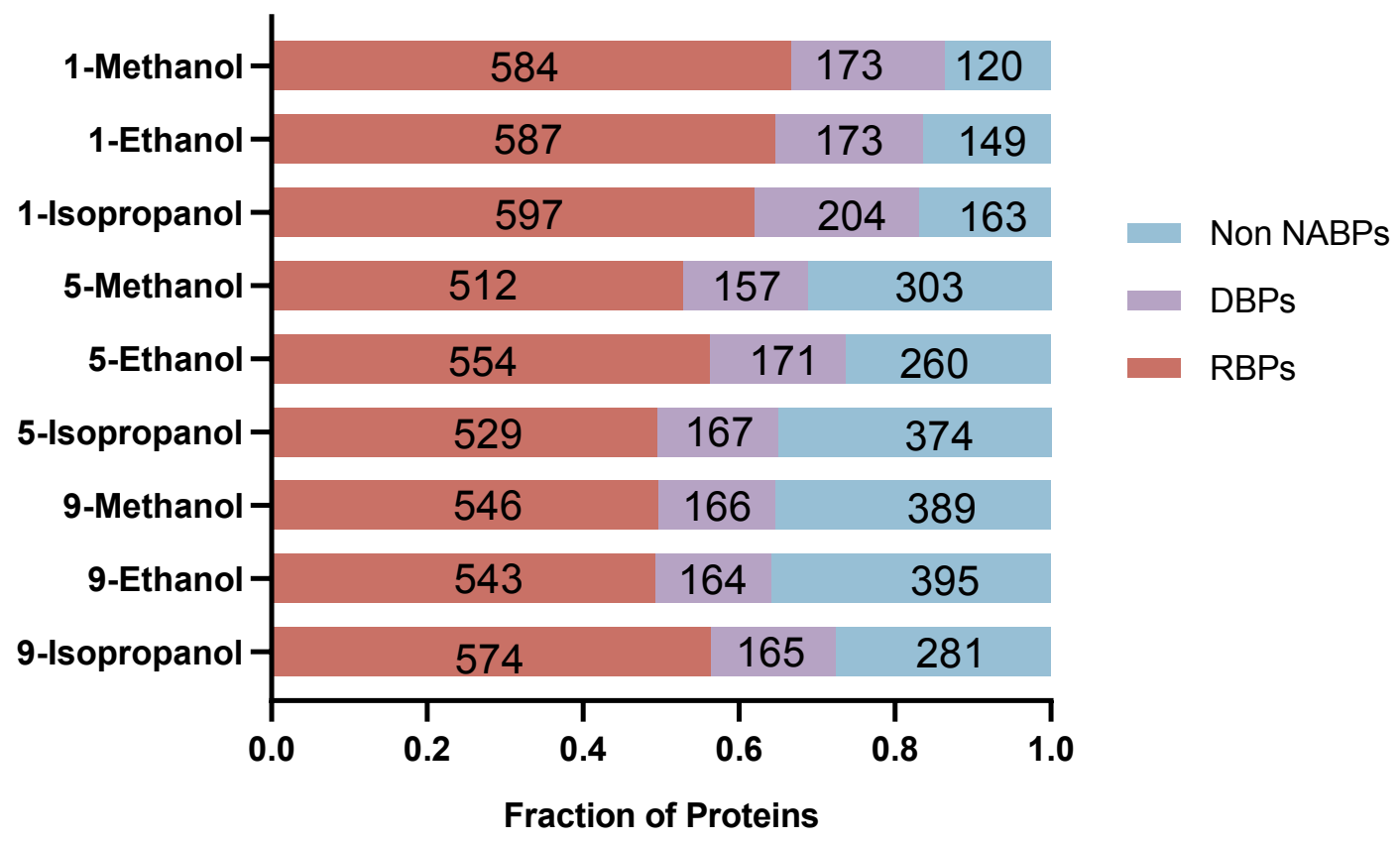

Figure S5. The number and proportion of GO annotated DBPs, RBPs and Non-NABPs obtained with different precipitants and dosage 


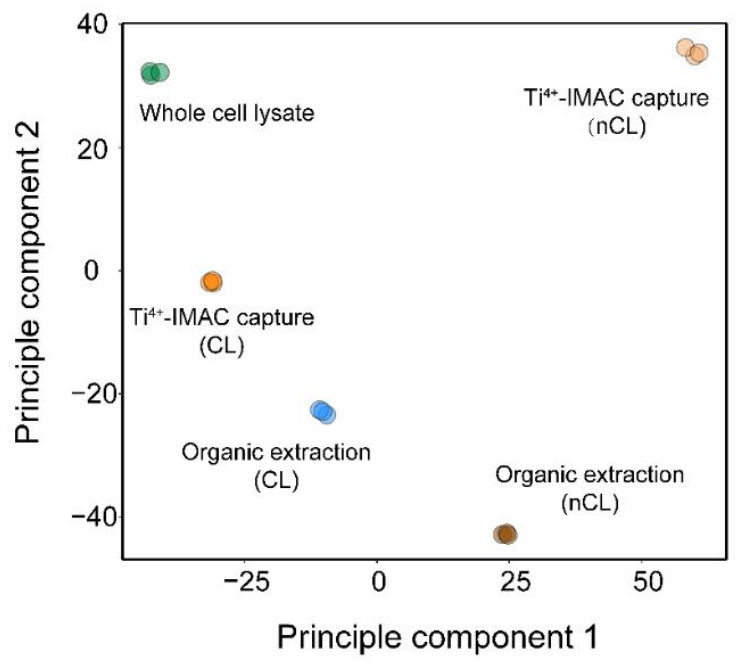

Figure S6. Principal component analysis of the quantification data of proteins in different groups.

(A)

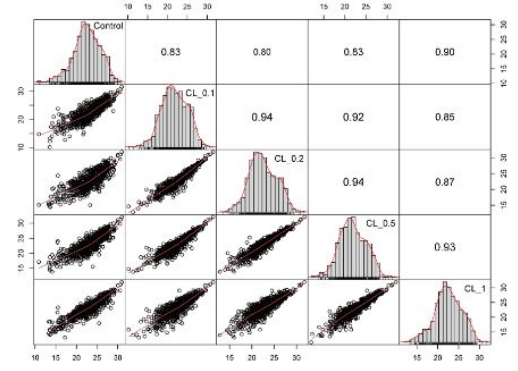

(C)

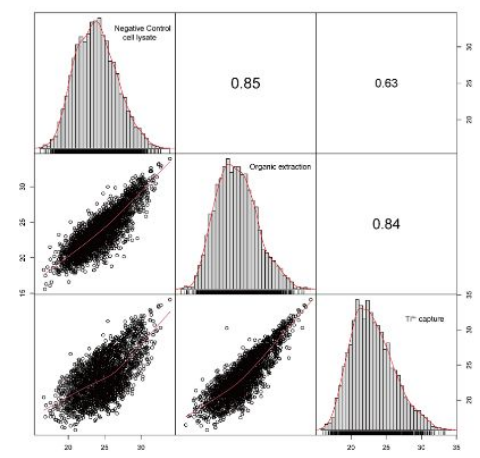

(B)

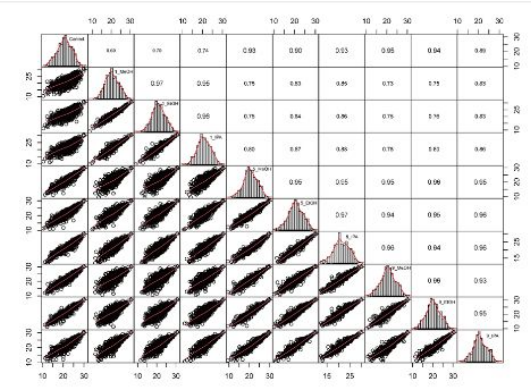

(D)

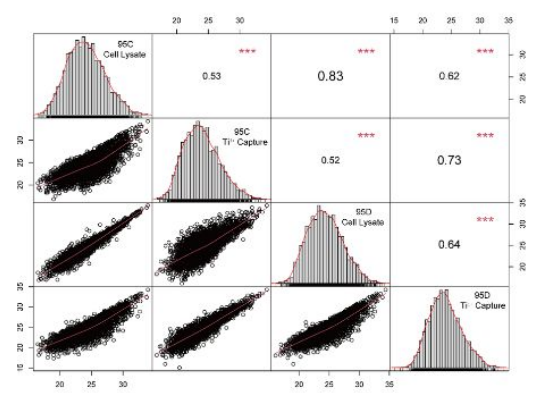

Figure S7. Pearson coefficients of the quantitative analysis of proteins obtained from (A) Formaldehyde concentration optimization, (B) organic solvent pretreatment optimization, (C) NAs-protein complexes enrichment method comparison, and (D) application of $\mathrm{Ti}^{4+}$-IMAC method in Lung cancer cells. 

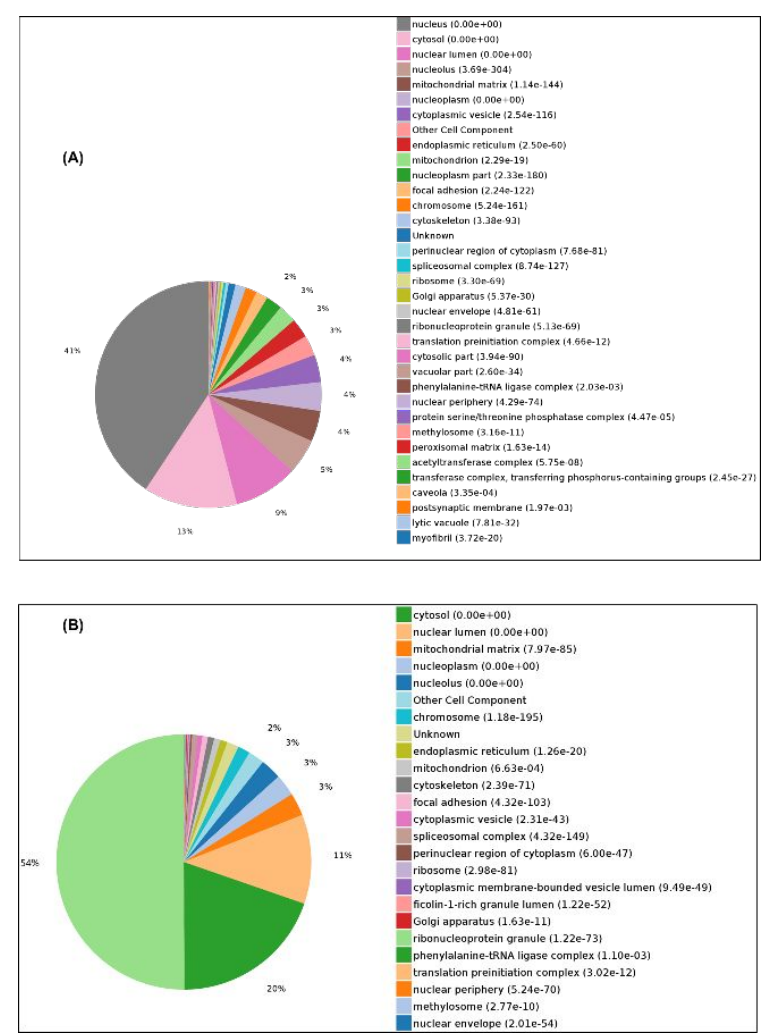

Figure S8. (A) Cell components analysis of protein extracted by organic solvent extraction. (B) Cell components analysis of protein extracted by $\mathrm{Ti}^{4+}-\mathrm{IMAC}$ capture method. 
(A)

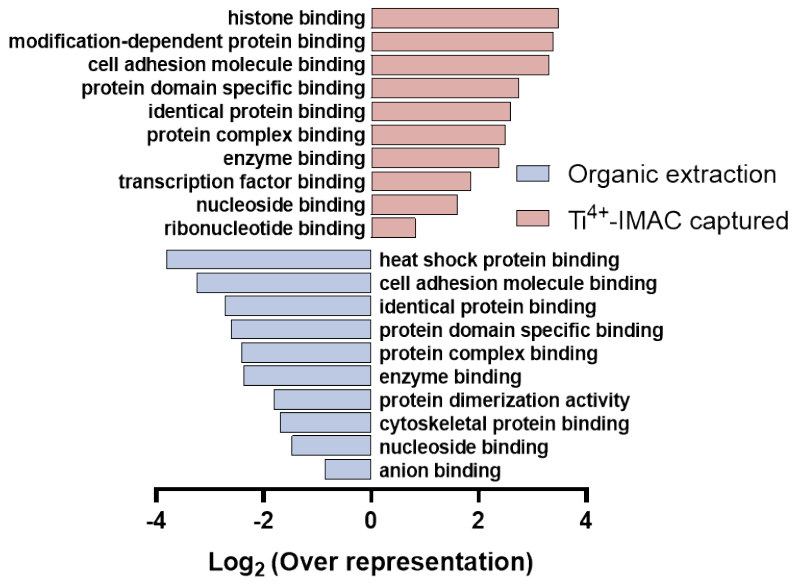

(B)

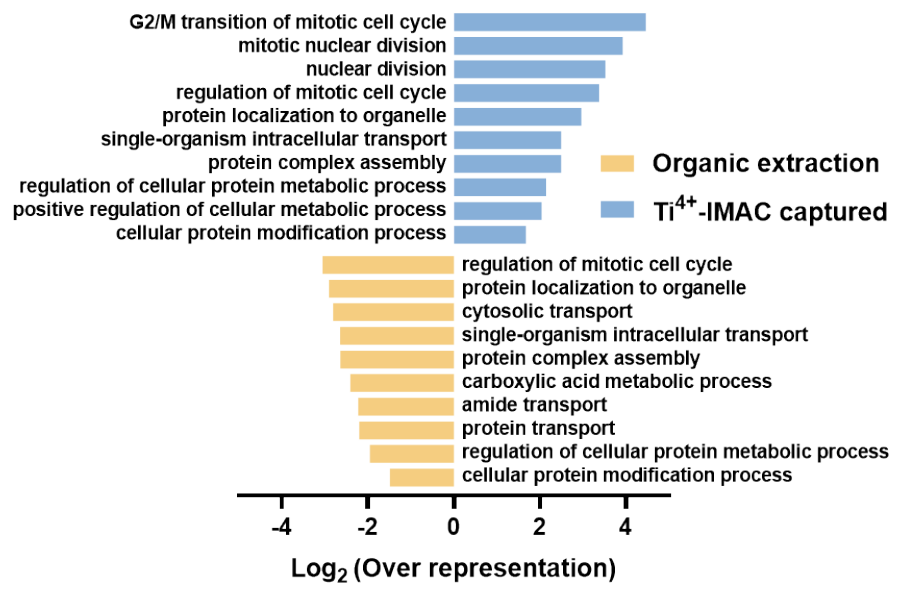

Figure S9. (A) Ten of the most significant over-represented molecular function GO terms of obtained from 1255 non-NABP proteins in organic solvent extraction and 767 non-NABP proteins in $\mathrm{Ti}^{4+}-$ IMAC method. (B) Ten of the most significant over-represented biological process GO terms of proteins obtained from 1255 non-NABP proteins in organic solvent extraction and 767 non-NABP proteins in $\mathrm{Ti}^{4+}$-IMAC capture method. 
(A)

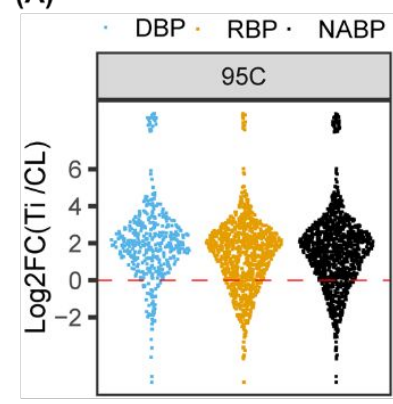

(B)

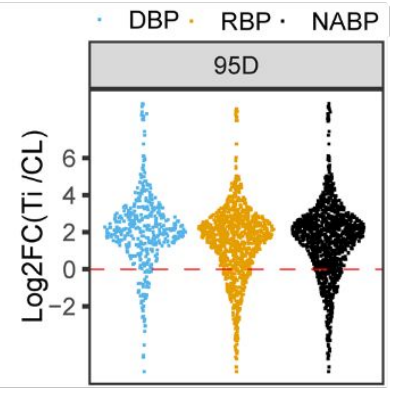

(C)

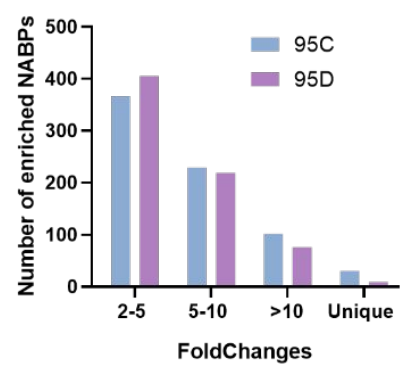

Figure S10. The fold change distribution of DBPs, RBPs and NABPs in (A) 95C and (B) 95D ( $\mathrm{Ti}^{4+}-$ IMAC capture method/Total cell lysate). (C) The number of NABPs significantly enriched in $95 \mathrm{C}$ and $95 \mathrm{C}\left(\mathrm{Ti}^{4+}-\right.$ IMAC capture method/Total cell lysate). 